\title{
Retzius Sparing (RS) Robotic Assisted Radical Prostatectomy (RARP) and Retzius Space Reconstruction Technique after RARP Improve Urinary Continence Compared to Conventional RARP (C-RARP): Systematic Review and Metanalysis
}

\section{William Pertuz Genes ${ }^{1 *}$, Maria Claudia bicudo ${ }^{1}$, Pablo Miranda Machado², Pericles Rios Auad ${ }^{3}$, Liseth Echavez Pacheco ${ }^{4}$, Hamilton de Campos Zampolli, Igor Nunes da Silva ${ }^{3}$, Daniel Coser Gomes ${ }^{5}$ and Marcos Tobias-Machado ${ }^{1}$}

${ }^{1}$ Departamento de Urologia, Faculdade de Medicina do ABC, Santo André, São Paulo, Brazil

${ }^{2}$ Universidad Metropolitana, Universidad Nacional de Colombia, Colombia

${ }^{3}$ Instituto do Câncer Arnaldo Vieira de Carvalho, São Paulo, Brazil

${ }^{4}$ Corporación Universitaria Rafael Núñez, Cartagena, Colombia

${ }^{5}$ Hospital Municipal Dr. Jose de Carvalho Florence, Brazil

*Corresponding author: William Pertuz Genes, Departamento de Urologia, Faculdade de Medicina do ABC, Av. Principe de Gales, $n^{\circ} 821$, Santo André, São Paulo, 09060-650, Brazil, Tel: +55-1196845878

\begin{abstract}
Objectives: Several parameters are important to evaluate good oncological and functional results after robotic prostatectomy. We hypothesize that preservation or reconstruction of Retzius space can be valuable for better results outcomes, especially in terms of early continence recovery.

Methods: In October 2018, electronic searches were conducted in the following databases: Cochrane, EMBASE, PubMed and Google Scholar. Manual searches in the reference lists of all the reviews and the relevant studies were identified. Searches of abstracts of relevant congresses were made. To locate additional studies, experts in the field were contacted (Supplementary Table 1).

Randomized controlled trials and observational studies comparing RS X anterior approach or RSR X anterior approach for the treatment of patients with localized prostate cancer were selected.

Results: We included 8 studies: Two randomized controlled clinical trials, three retrospective observational studies and three prospective observational studies.
\end{abstract}

Continence recovery: In the RS-RARP group, the overall continence recovery in 1-2 weeks or 10 days or immediately after surgery was significantly faster compared to the conventional anterior approach (random effect (RE): OR = 4.17; 95\% Cl: 2.14-8.14; $p<0.0001)$.

Positive surgical margins (PSMs): Between the two groups in both stages $\mathrm{pT} 2$, there were no significant differences (RE: OR $=1.06 ; 95 \% \mathrm{Cl}: 0.62-1.81 ; p<0.83$ and $\mathrm{pT} 3$ stages (RE: OR = 1.33; 95\% Cl: 0.66-2.66; $\mathrm{p}<0.42$ ).

Complication rate: Between RS group and conventional anterior approach, there were no significant differences (RE: OR $=0.98 ; 95 \% \mathrm{Cl}: 0.51-1.90 ; p<0.96$ ). In the RS group compared to the previous conventional, the urinary function (IPSS) was significantly higher.

Conclusions: The RS-RARP group had a faster continence recovery compared to C-RARP, while we found no difference in terms of long-term urinary function or oncological outcomes.

\section{Introduction}

Prostate cancer is the leading cause of death among men in industrialized countries due to oncological caus-

Citation: Genes WP, Bicudo MC, Machado PM, Auad PR, Pacheco LE, et al. (2019) Retzius Sparing (RS) Robotic Assisted Radical Prostatectomy (RARP) and Retzius Space Reconstruction Technique after RARP Improve Urinary Continence Compared to Conventional RARP (C-RARP): Systematic Review and Metanalysis. Int Arch Urol Complic 5:065. doi.org/10.23937/2469-5742/1510065

Accepted: November 23, 2019: Published: November 25, 2019

Copyright: (C) 2019 Genes WP, et al. This is an open-access article distributed under the terms of the Creative Commons Attribution License, which permits unrestricted use, distribution, and reproduction in any medium, provided the original author and source are credited. 
es and prostate-specific antigen (PSA) screening for it is common in many countries around the world [1] is the second most common cancer and the sixth cause of cancer in men [2]. The presence of negative surgical margins, continence and erectile dysfunction are the most relevant issues for patients undergoing RARP [3].

The "pentafecta" outcomes defined the best possible scenario for radical prostatectomy. Consisting in: Absence of early complications, no positive surgical margins (PSM), continence recovery, sexual potency and absence of biochemical recurrence [4].

At the beginning of 2010, Galfano, et al. described the Retzius Sparing (RS) procedure, which allows the possibility of performing completely intrafascial prostatectomies. This technique retains the complete anatomical integrity of the "Aphrodite's veil", which contains the neurovascular bundles. Good functional results can be expected in patients undergoing a high intrafascial procedure, since several studies report the presence of nerve structures within the higher characteristics of the Aphrodite veil and the endopelvic fascia.

With the standard RALP procedure as well as with intrafascial approaches preserving Aphrodite's veil, the higher aspect of the veil has to be opened. In contrast, with the Bocciardi approach, the veil does not have to be opened at any point, providing a strong rationale for obtaining better results [5]. The preservation of the bladder neck, the preservation of the prostatic fascia among other reconstructive techniques and the total anatomical reconstruction seems to improve continence [6]. Other techniques related to the development of continence are the complete reconstruction of the Retzius space, also reducing the early and late perioperative complications [7].

The technique Retzius-Sparing (RS-RARP) in which the approach is posterior and is completely intrafascial, technically more challenging than the conventional technique, in this approach the parietal peritoneum is incised for $5-7 \mathrm{~cm}$ on the anterior surface of the Douglas pouch. The seminal vesicles and the vas deferens are isolated. An intrafascial plane is maintained (with complete nerve preservation whenever possible and onco- logically safe). In case of adhesions, palpable disease, the surgeon will choose to follow a wider dissection plan. The vesicoprostatic junction is recognized, isolated and sectioned; the bladder neck is preserved when reasonably safe. The forward surface of the prostate is blunt dissected and isolated from the Santorini plexus without incision. The apex isolation is completed and the urethra is incised. The anastomosis is performed using a adapted Van Velthoven suture from 12 o'clock. This technique results in the preservation of all structures located in the Retzius space, such as; endopelvic fascia, Santorini plexus, pubovesical ligaments and accessory pudendal arteries. Rates of complications are relatively comparable and continence is quicker [8].

In the reconstructive technique (RSR), the Retzius space is opened, dissection of the bladder is performed, conventional technique of robotic prostatectomy is performed, the endopelvic fascia is opened and the dorsal venous complex is secured, neurovascular bundle preservation, prostate removal, anastomosis and subsequent closure of the Retzius space, closing the peritoneum thus relocating the bladder to the anterior abdominal wall and, the continence recovery was significantly faster in the RSR group compared to patients without reconstruction [9].

We led this meta-analysis and review of the literature. Evaluate the effectiveness and the postoperative outcomes of radical prostatectomy assisted by the robot that retains Retzius (RS-RARP) and Retzius space reconstruction technique (RSR-RARP) compared to conventional technique (C-RARP).

\section{Objectives}

To evaluate the efficacy, effectiveness, safety and clinical, functional and oncological outcomes of Retzius sparing robot-assisted radical prostatectomy (RS-RARP) and Retzius space reconstruction technique (RSR-RARP) in comparison with the conventional or standard RARP (C-RARP) (Table 1).

\section{Methods}

\section{Inclusion criteria for considering studies for this review}

Table 1: Summary of all studies about Retzius-sparing robot-assisted radical prostatectomy.

\begin{tabular}{|l|l|l|l|l|l|l|}
\hline First author \& year & Study design & Participants & RS-RARP & C-RARP & Mean age (years) & Risk of bias \\
\hline Chang 2018 [21] & Case-control & 60 & 30 & 30 & 66.2 & Unclear \\
\hline Dalela 2017 [18] & RCT & 120 & 60 & 60 & 61.2 & Low \\
\hline Eden 2017 [20] & Retrospective & 80 & 40 & 40 & 64 & Unclear \\
\hline Ghanem 2017 [9] & Prospective & 102 & 51 & 51 & 61.9 & Unclear \\
\hline Kiat Lim 2014 [12] & Retrospective & 100 & 50 & 50 & 65.9 & Unclear \\
\hline Menon 2017 [11] & RCT & & & & Low \\
\hline Sayyid 2017 [22] & Prospective & 200 & 100 & 100 & 61.5 & Unclear \\
\hline Tobias Machado 2017 [26] & Prospective & 60 & 30 & 30 & 63.9 & Unclear \\
\hline
\end{tabular}

$\mathrm{RCT}=$ Randomised Controlled Trial; RS-RARP = Retzius-Sparing; C-RARP = Conventional Approach. 


\section{Types of studies:}

1) Randomized controlled trials.

2) Comparative observational studies (including case series).

\section{Types of participants:}

Patients with low-intermediate risk prostate cancer according to NCCN (National Comprehensive Cancer Network) Guidelines.

\section{Types of interventions:}

1) RS-RARP or posterior RARP.

2) Retzius space reconstruction RSR-RARP.

3) C-RARP or standard RARP or anterior RARP.

\section{Types of outcome measures:}

\section{Primary outcomes:}

1) Continence recovery:

-Overall continence recovery at 1-2 week or 10 days or immediate post-op.

-Overall continence recovery at 4 weeks post-op.

-Overall continence recovery at 3 months post-op.

2) Positive surgical margins (PSMs):

-PSMs pT2.

-PSMs pT3.

3) Complications:

Complication rates.

4) Urinary function:

IPSS score at 3 months. Post-operation

\section{Secondary outcomes:}

1) Continence recovery:

-Overall continence recovery at 6 months post-op.

-Overall continence recovery at 12 months post-op.

-1 Pads/day at 4 weeks post-op.

-Time to urinary continence recovery.

2) Complications:

-Median operating time.

-Estimated blood loss.

-Length of stay.

\section{Search methods for identification of studies:}

Electronic searches: In October 2018, electronic searches were conducted in the following databases: Cochrane, EMBASE, PubMed and Google Scholar using the terms "Retzius-sparing", "posterior approach", and "robot assisted radical prostatectomy" were performed to identify studies published up to October 2018, com- paring RS-RARP to C-RARP.

Searching other resources: Searches of abstracts of relevant congresses were made.

\section{Data collection and analysis}

Selection of studies: PM and WP independently selected potentially relevant studies according to previously defined selection criteria. Disagreements were resolved by consensus.

Data extraction and management: After defining the studies to include and exclude by consensus, the following data were extracted from each study: First author, year of publication, study design, participants, interventions, average age of the participants, risk of bias, results and notes.

Assessment of risk of bias in included studies: To evaluate the risk of bias in the selected studies, we applied the tools of the Cochrane Collaboration, which assesses the risk of bias in six domains: Generation of sequences, concealment of allocation, blinding, incomplete results data, selective results reports and other potential biases.

Measures of treatment effect: For statistical analysis, we use Review Manager 5.3 provided by the Cochrane Collaboration. We summarized the dichotomous and continuous results in terms of odds ratios (OR) and with a standardized mean difference (SMD) with their 95\% confidence intervals, respectively.

\section{Assessment of heterogeneity}

Among the trials that used the chi-squared statistic, heterogeneity was examined with a significance level of 0.10 and the 12 statistic. We considered moderate or high levels of heterogeneity at the 12 estimation above $50 \%$ and a sensitivity analysis was performed.

Assessment of reporting biases: We used a broad search strategy that included the evaluation of published and unpublished studies in order to reduce the risk of publication bias.

Data synthesis: The results were grouped when the interventions and populations had similarities and the differences were estimated using fixed and random effects models. In the absence of significant differences between the two models, the final results are presented using random effects models.

\section{Subgroup analysis and investigation of heteroge- neity}

Sensitivity analysis: A sensitivity analysis was done in cases where the results were heterogeneous.

\section{Results}

\section{Description of studies}

We included 8 studies: Two randomized controlled 
clinical trials, three retrospective observational studies and three prospective observational studies. We observed a sufficient similarity between the parameters in the studies. In all the studies, the RS-RARP and conventional procedures were compared. Dichotomous vari- ables (recovery of continence, PSMs and complication rates) were comparable for the meta-analysis. The only study that reported data and results about recovery of potency after treatment with RS-RARP was Menon, et al. (See the Figure 1 Study flow diagram).

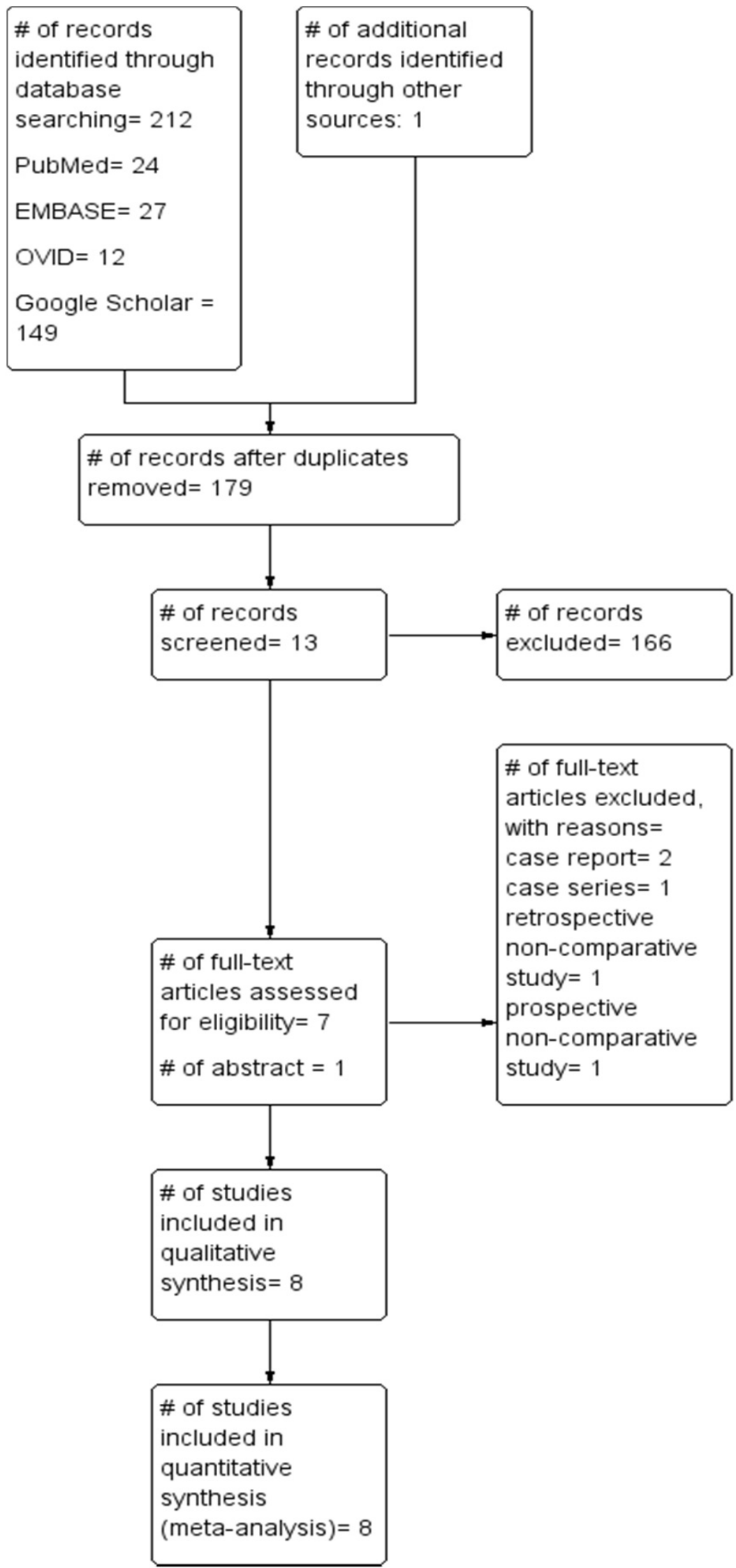

Figure 1: Study flow diagram. 


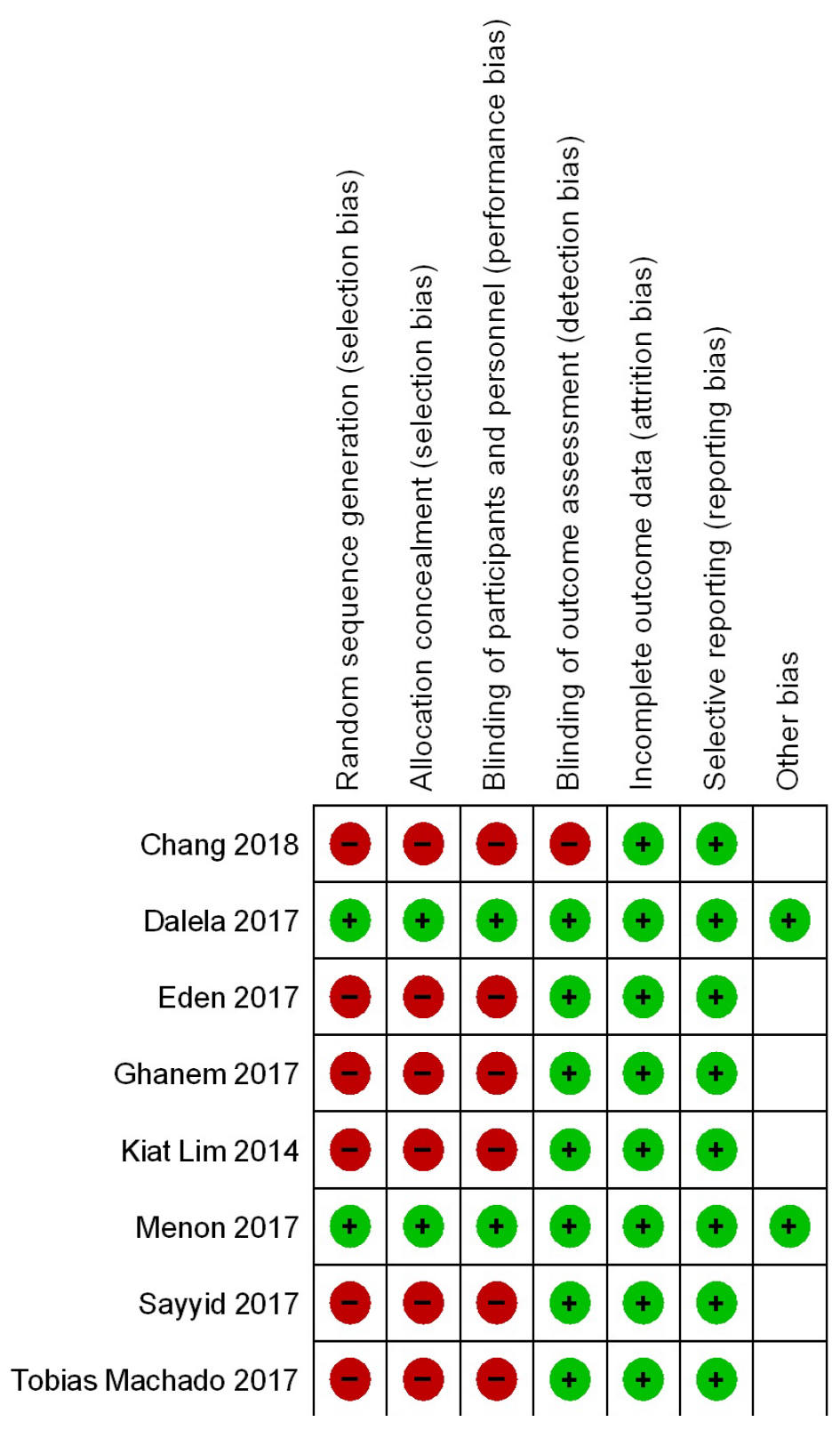

Figure 2: Risk of bias graph: Review authors' judgements about each risk of bias item presented as percentages across all included studies.

Random sequence generation (selection bias)

Allocation concealment (selection bias)

Blinding of participants and personnel (performance bias)

Blinding of outcome assessment (detection bias) Incomplete outcome data (attrition bias)

Selective reporting (reporting bias)

Other bias
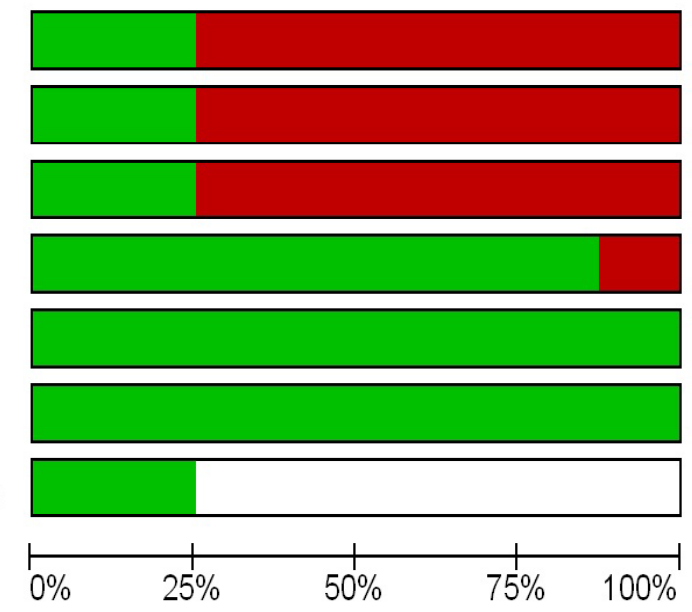

Low risk of bias

Unclear risk of bias

High risk of bias

Figure 3: Risk of bias summary: Review authors' judgements about each risk of bias item for each included study. 


\section{Results of the search}

Included studies: After reviewing 179 articles we determined that 8 studies met our inclusion criteria.

Excluded studies: We excluded 171 articles from the systematic review, for reasons such as the following: There was no comparison group, case report or series of cases, descriptive studies or narrative reviews.

Risk of bias in included studies: See Figure 2 Risk of bias graph and Figure 3 Risk of bias summary.

\section{Effects of interventions}

Continence Recovery: In the RS group, the overall continence recovery in 1-2 weeks or 10 days or immediately after the surgery (random effect (RE): $O R=4.17$;
95\% Cl: 2.14-8.14; $p<0.0001$, see Figure 4) was significantly faster and higher, respectively, compared to the conventional anterior approach.

Similar results were found In the RS group, the overall continence recovery at 4 weeks post-op. after surgery (random effect (RE): OR $=4.61 ; 95 \% \mathrm{Cl}: 2.72-7.81$; $\mathrm{p}<0.00001$, See Figure 5), In the RS group, the overall continence recovery at 3 months post-op. after surgery (random effect (RE): $\mathrm{OR}=3.32 ; 95 \% \mathrm{Cl}: 2.07-5.32 ; \mathrm{p}<$ 0.00001 , see Figure 6).

Overall continence recovery at 6 months post-op. after surgery (random effect (RE): $\mathrm{OR}=3.70 ; 95 \% \mathrm{Cl}: 2.33$ $5.88 ; p<0.00001$, See Figure 7), In RS group, the overall continence recovery at 12 months post-op. after sur-

\begin{tabular}{|c|c|c|c|c|c|c|c|c|c|c|}
\hline \multirow{2}{*}{$\begin{array}{l}\text { Study or Subgroup } \\
\text { Chang } 2018\end{array}$} & \multicolumn{2}{|c|}{ RS-RARP } & \multicolumn{2}{|c|}{ C-RARP } & Weight & $\begin{array}{c}\text { Odds Ratio } \\
\text { M-H, Random, } 95 \% \mathrm{Cl}\end{array}$ & \multicolumn{4}{|c|}{$\begin{array}{c}\text { Odds Ratio } \\
\text { M-H, Random, } 95 \% \mathrm{Cl}\end{array}$} \\
\hline & 20 & 30 & 8 & 30 & $18,9 \%$ & $5,50[1,81,16,68]$ & & & & \\
\hline Dalela 2017 & 46 & 60 & 36 & 60 & $25,5 \%$ & $2,19[0,99,4,83]$ & & & & \\
\hline Eden 2017 & 37 & 40 & 27 & 40 & $15,1 \%$ & $5,94[1,54,22,90]$ & & & & \\
\hline Kiat Lim 2014 & 35 & 50 & 25 & 50 & $24,8 \%$ & $2,33[1,03,5,30]$ & & & & \\
\hline Tobias Machado 2017 & 26 & 30 & 9 & 30 & $15,7 \%$ & $15,17[4,09,56,25]$ & & & & \\
\hline Total $(95 \% \mathrm{Cl})$ & & 210 & & 210 & $100,0 \%$ & $4,17[2,14,8,14]$ & & & & \\
\hline Total events & 164 & & 105 & & & & & & & \\
\hline $\begin{array}{l}\text { Heterogeneity: } \operatorname{Tau}^{2}=0 \text {, } \\
\text { Test for overall effect: } Z\end{array}$ & $\begin{array}{l}29 ; \mathrm{Ch}^{2}= \\
=4,19(\mathrm{P}\end{array}$ & $\begin{array}{l}8,32,0 \\
<0.000\end{array}$ & $\begin{array}{l}\text { ff }=4(P= \\
1)\end{array}$ & 0,08); & $P^{2}=52 \%$ & & 0.01 & $\begin{array}{c}0.1 \\
\text { Favours [C-RARP] }\end{array}$ & $\begin{array}{cc}10 \\
\text { Favours [RS-RARP] }\end{array}$ & 100 \\
\hline
\end{tabular}

Figure 4: Forest plot of comparison: Continence recovery, outcome: Overall continence recovery at 1-2 week or 10 days or immediate post-op.

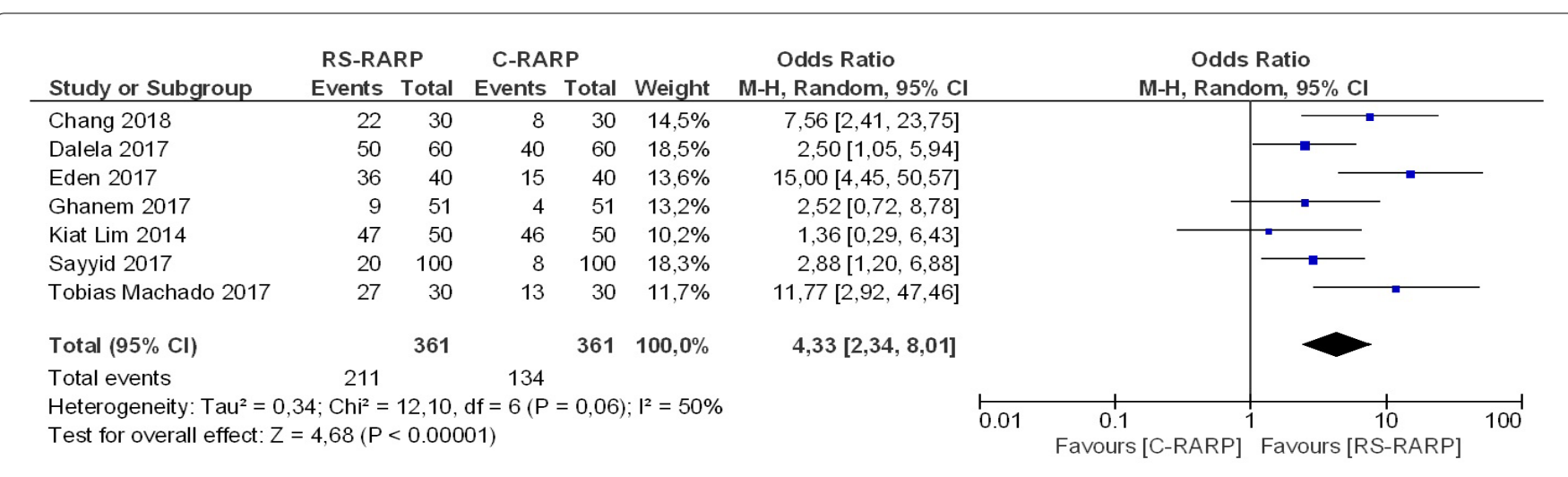

Figure 5: Forest plot of comparison: Continence recovery, outcome: Overall continence recovery at 4 weeks post-op.

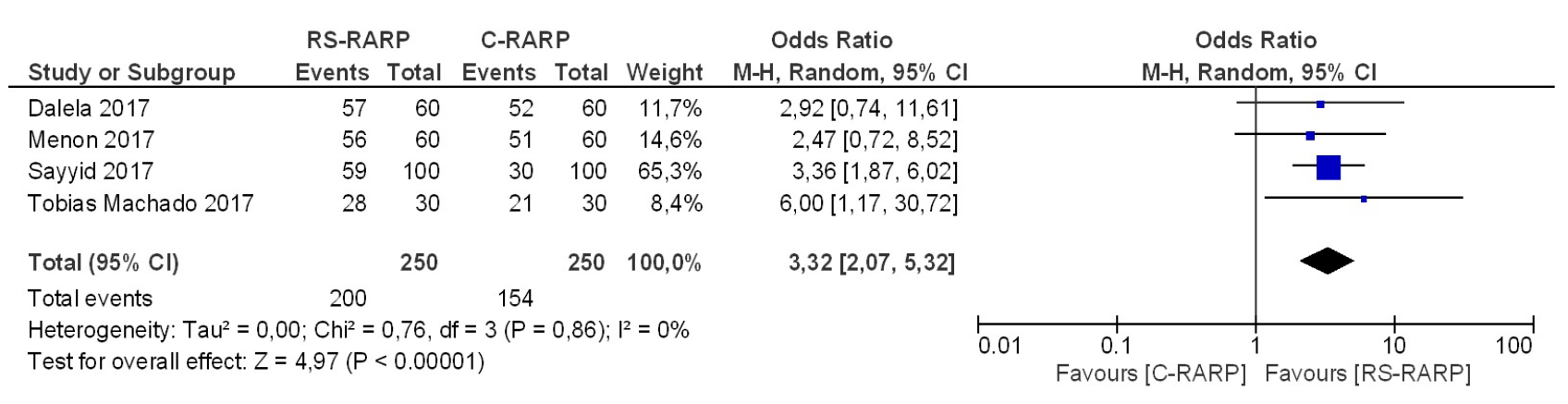

Figure 6: Forest plot of comparison: Continence recovery, outcome: Overall continence recovery at 3 months post-op. 


\begin{tabular}{|c|c|c|c|c|c|c|c|c|c|c|}
\hline \multirow{2}{*}{$\begin{array}{l}\text { Study or Subgroup } \\
\text { Ghanem } 2017\end{array}$} & \multicolumn{2}{|c|}{ RS-RARP } & \multicolumn{2}{|c|}{ C-RARP } & Weight & $\begin{array}{c}\text { Odds Ratio } \\
\text { M-H, Random, } 95 \% \mathrm{Cl}\end{array}$ & \multicolumn{4}{|c|}{$\begin{array}{c}\text { Odds Ratio } \\
\text { M-H, Random, } 95 \% \mathrm{Cl}\end{array}$} \\
\hline & 44 & 51 & 34 & 51 & $25,5 \%$ & $3,14[1,17,8,44]$ & & & $\longrightarrow$ & \\
\hline Menon 2017 & 59 & 60 & 56 & 60 & $5,0 \%$ & $4,21[0,46,38,86]$ & & & & \\
\hline Sayyid 2017 & 81 & 100 & 50 & 100 & $61,6 \%$ & $4,26[2,26,8,04]$ & & & $\rightarrow$ & \\
\hline Tobias Machado 2017 & 28 & 30 & 26 & 30 & $7,8 \%$ & $2,15[0,36,12,76]$ & & & & \\
\hline Total $(95 \% \mathrm{CI})$ & & 241 & & 241 & $100,0 \%$ & $3,74[2,27,6,15]$ & & & & \\
\hline Total events & 212 & & 166 & & & & & & & \\
\hline $\begin{array}{l}\text { Heterogeneity: } \mathrm{Tau}^{2}=0 \\
\text { Test for overall effect: } Z\end{array}$ & $\begin{array}{l}0 ; \mathrm{Chi}^{2}= \\
5,18(\mathrm{P}\end{array}$ & $\begin{array}{l}0,66, d \\
<0.000\end{array}$ & $\begin{array}{l}\text { ff }=3(P= \\
01)\end{array}$ & $0,88)$ & $1^{2}=0 \%$ & & 0.01 & $\begin{array}{c}0.1 \\
\text { Favours [C-RARP] }^{1}\end{array}$ & $\begin{array}{c}10 \\
\text { Favours [RS-RARP] }\end{array}$ & 100 \\
\hline
\end{tabular}

Figure 7: Forest plot of comparison: Continence recovery, outcome: Overall continence recovery at 6 months post-op.

\begin{tabular}{|c|c|c|c|c|c|c|c|c|c|c|}
\hline \multirow[b]{2}{*}{ Study or Subgroup } & \multicolumn{2}{|c|}{ RS-RARP } & \multicolumn{2}{|c|}{ C-RARP } & \multirow[b]{2}{*}{ Weight } & \multirow{2}{*}{$\begin{array}{c}\text { Odds Ratio } \\
\mathrm{M}+\mathrm{H}, \text { Random, } 95 \% \mathrm{Cl}\end{array}$} & \multirow{2}{*}{\multicolumn{4}{|c|}{$\begin{array}{c}\text { Odds Ratio } \\
\mathrm{M}-\mathrm{H}, \text { Random, } 95 \% \mathrm{Cl}\end{array}$}} \\
\hline & Events & Total & Events & Total & & & & & & \\
\hline Chang 2018 & 30 & 30 & 28 & 30 & $11,3 \%$ & $5,35[0,25,116,31]$ & & & & \\
\hline Ghanem 2017 & 47 & 51 & 47 & 51 & $26,0 \%$ & $1,00[0,24,4,24]$ & & & & \\
\hline Menon 2017 & 59 & 60 & 56 & 60 & $17,3 \%$ & $4,21[0,46,38,86]$ & & & & \\
\hline Sayyid 2017 & 97 & 100 & 68 & 100 & $28,9 \%$ & $15,22[4,48,51,71]$ & & & - & \\
\hline Tobias Machado 2017 & 29 & 30 & 27 & 30 & $16,4 \%$ & $3,22[0,32,32,89]$ & & & & \\
\hline Total $(95 \% \mathrm{Cl})$ & & 271 & & 271 & $100,0 \%$ & $4,13[1,22,13,98]$ & & & & \\
\hline Total events & 262 & & 226 & & & & & & & \\
\hline $\begin{array}{l}\text { Heterogeneity: } \operatorname{Tau}^{2}=0 \\
\text { Test for overall effect: } Z\end{array}$ & 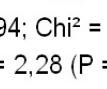 & $\begin{array}{r}8,27, d \\
=0,02)\end{array}$ & $f=4(P=$ & $=0,08)$ & $l^{2}=52 \%$ & & 0.01 & $\begin{array}{c}0.1 \\
\text { Favours [C-RARP] }\end{array}$ & $\begin{array}{cc}1 & 10 \\
\text { Favours [RS-RARP] }\end{array}$ & 100 \\
\hline
\end{tabular}

Figure 8: Forest plot of comparison: Continence recovery, outcome: Overall continence recovery at 12 months post-op.

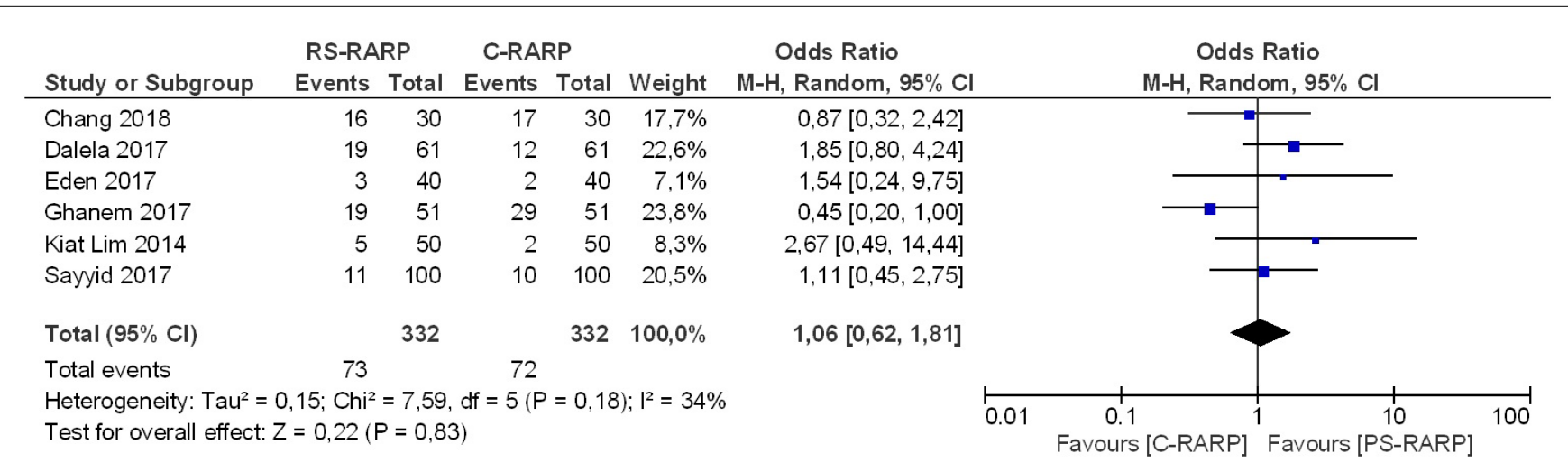

Figure 9: Forest plot of comparison: Positive surgical margins (PSMs), outcome: PSMs pT2.

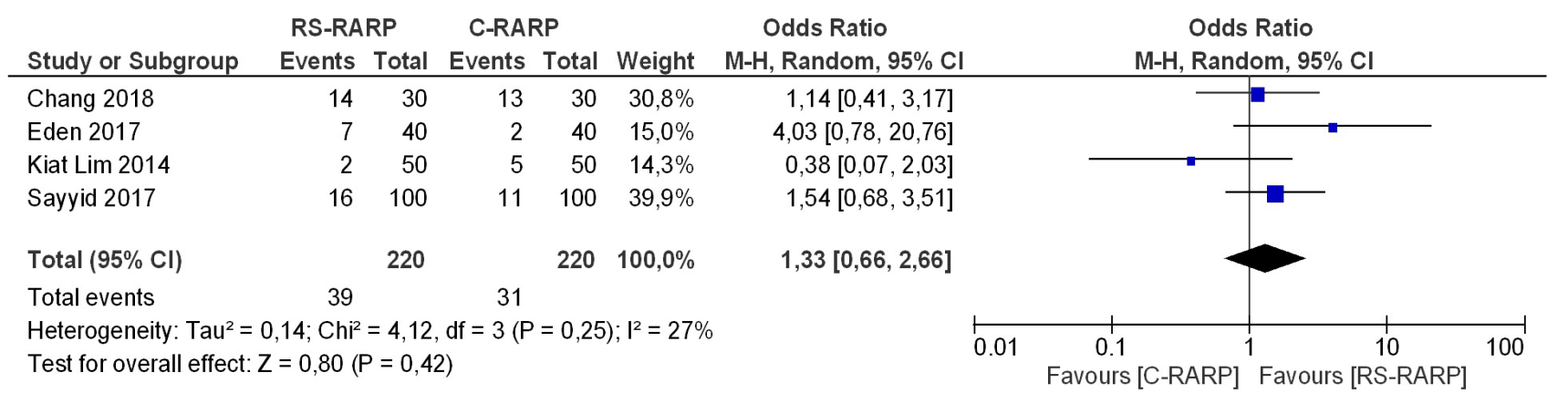

Figure 10: Forest plot of comparison: Positive surgical margins (PSMs), outcome: PSMs pT3.

gery was significantly faster compared to conventional anterior approach (random effect (RE): OR = 3.66; $95 \%$ Cl: 1.17-11.46; $p<0.03$, See Figure 8).

\section{Positive Surgical Margins (PSMs)}

There were no significant differences between the two groups in both stages pT2 (RE: OR $=1.06 ; 95 \% \mathrm{Cl}$ : $0.62-1.81 ; p<0.83$, See Figure 9) and pT3 stages (RE: OR = 1.33; 95\% Cl: 0.66-2.66; $\mathrm{p}<0.42$, See Figure 10).

\section{Complication rate}

There were no significant differences between RS 


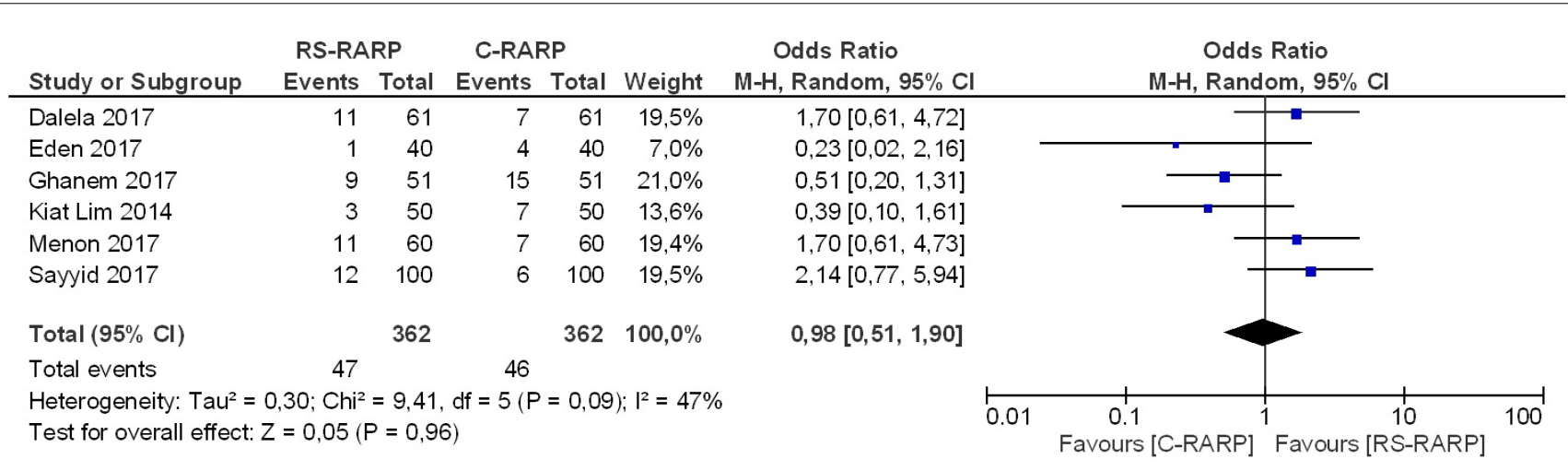

Figure 11: Forest plot of comparison: Complications, outcome: Complication rates.

\begin{tabular}{|c|c|c|c|c|c|c|c|c|c|}
\hline \multirow[b]{2}{*}{ Study or Subgroup } & \multicolumn{3}{|c|}{ RS-RARP } & \multicolumn{3}{|c|}{ C-RARP } & \multicolumn{2}{|r|}{ Std. Mean Difference } & \multirow{2}{*}{$\begin{array}{r}\text { Std. Mean Difference } \\
\text { IV, Random, } 95 \% \mathrm{Cl}\end{array}$} \\
\hline & Mean & SD & Total & Mean & SD & Total & Weight & IV, Random, $95 \% \mathrm{Cl}$ & \\
\hline Chang 2018 & 211,83 & 49,97 & 30 & 214 & 48,8 & 30 & $50,2 \%$ & $-0,04[-0,55,0,46]$ & \\
\hline Kiat Lim 2014 & 117 & 4 & 50 & 141 & 5 & 50 & $49,8 \%$ & $-5,26[-6,10,-4,42]$ & $\mathbf{\square}$ \\
\hline Total $(95 \% \mathrm{Cl})$ & & & 80 & & & 80 & $100,0 \%$ & $-2,64[-7,75,2,47]$ & \\
\hline \multicolumn{9}{|c|}{$\begin{array}{l}\text { Heterogeneity: } \text { Tau }^{2}=13,48 ; \mathrm{Chi}^{2}=108,55, \mathrm{df}=1(\mathrm{P}<0.00001) ; \mathrm{I}^{2}=99 \% \\
\text { Test for overall effect: } Z=1,01(P=0,31)\end{array}$} & $\begin{array}{ccccc}1 & 1 & 1 & 1 & 1 \\
-20 & -10 & 0 & 10 & 20 \\
\text { Favours [C-RARP] } & \text { Favours [RS-R }\end{array}$ \\
\hline
\end{tabular}

Figure 12: Forest plot of comparison: Complications, outcome: Median operating time (min).

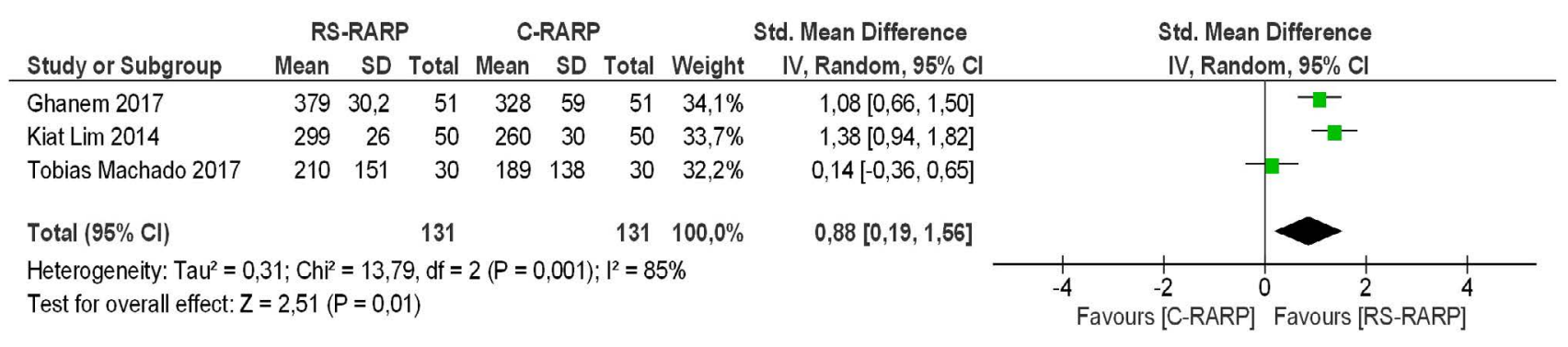

Figure 13: Forest plot of comparison: Complications, outcome: Estimated blood loss (ml).

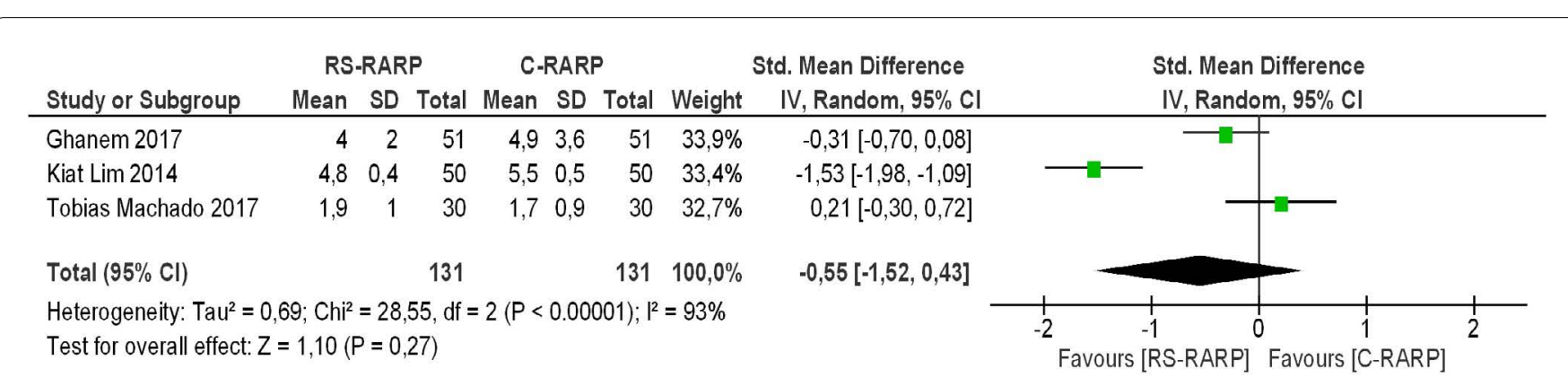

Figure 14: Forest plot of comparison: Complications, outcome: Length of stay (days).

group and conventional anterior approach (RE: OR = 0.98; 95\% Cl: 0.51-1.90; $\mathrm{p}<0.96$, See Figure 11). There were no significant differences in the median operating time, in the estimated blood loss and in the length of stay, see Figure 12, Figure 13 and Figure 14 respectively.

\section{Urinary Function}

We assessed urinary function with the International Prostate Symptom Score (IPSS) at 3 months post-operation, graduating patients with mild, moderate and severe symptoms as was significantly favourable in RS group compared to conventional approach (RE: SMD = -0.25 ; $95 \% \mathrm{Cl}$ : $-0.46--0.05$; $\mathrm{p}<0.01$, See Figure 15).

\section{Discussion}

In recent years, various techniques have been developed in order to achieve a faster continence. In patients under RARP, the fastest continence rates are reached at 2 to 6 months after surgery. The 2017 Annual Report of Intuitive Surgical Inc. stated that Da 


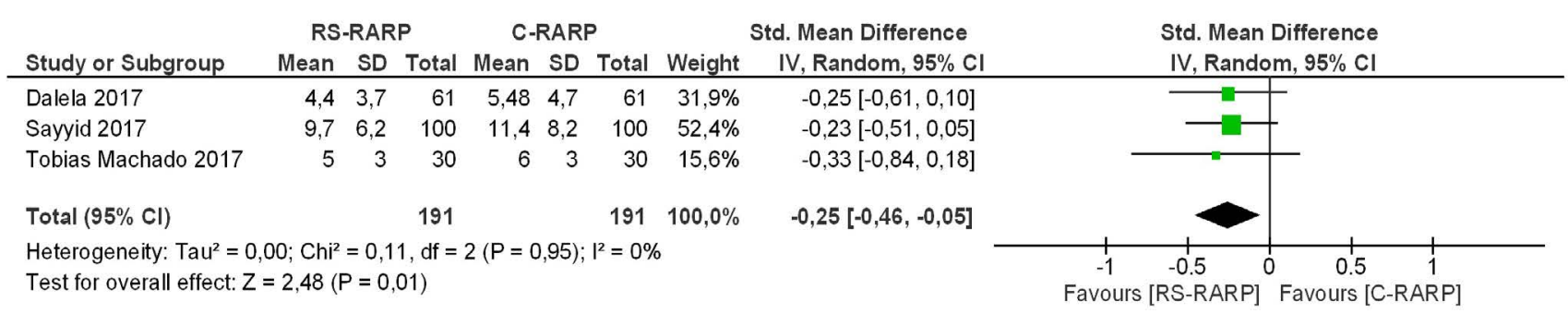

Figure 15: Forest plot of comparison: Urinary function, outcome: Mean IPSS score at 3 months.

Vinci prostatectomy had a significant increase in recent years [10]. The growth in the realization of this procedure motivates the need to innovate the techniques to improve specific results.

One of the limitations of the technique (RS-RALP) is the lack of global experience, therefore few preceptors to help teach this technique that is more challenging than the conventional one described at the beginning of this millennium, another limitation of the technique that It makes (RS-RALP) remain a little hidden is the small workspace that it offers compared to conventional technique, something that can make surgery difficult when the prostates are very large or very attached which sometimes obliges the surgeon to go for an extrafacial plane [9].

\section{Complications}

There were no statistically significant differences between the 2 techniques RS-RARP vs. C-RARP (RE: OR = 0.98, 95\% Cl: 0.51-1.90, p < 0.96), Galfano, et al. [5] in their first case report reported as complications 2 vesical perforations, in the second work of Galfano, et al. reported a complication due to prostate fossa bleeding which was re-operated, another patient with severe abdomen due to intestinal hernia through a lateral portal, another subject to embolization of a branch of the gluteal artery due to bleeding, 8 transfusions were required in group 1 and 4 in group 2 [8].

Concerning the work of Menon [11] the most common difficulty was symptomatic lymphocele that required percutaneous drainage, occurred in 9 of 120 patients (7.5\%), a patient with RS-RARP required reoperation, and a patient with C-RARP required reintervention 3 months later due to an umbilical hernia, no urethral stenosis was existent in any patient.

In the paper of Ghanem [9] which compares conventional technique with reconstruction of the extra-peritoneal space of Retzius shows that general complications do not diverge in the 2 groups, only the hernias in the portals were lower in the RS-RARP technique compared with C-RARP.

As stated by Lim, et al. [12] no significant differences were observed regarding the length of hospital stay, bleeding and intra- and post-operative complications.

We can say that the more we practice this technique the more experience we will have, which could possibly lead to fewer complications.

\section{PSM}

PSM are uniformly associated with an increased risk of biochemical and local disease, are considered as an adverse result that could be associated with the failure of the surgery, could be an indicator of recurrence, as well as the need for secondary treatment after RARP. 1 PSM can also cause significant psychological distress in men with commensurable PSMs compared to those who have negative margins [13-15]. Regarding biochemical recurrence $(B C R)$, they are unfavorable pathological findings as extra-prostatic extension and seminal vesicles or invasion of the bladder neck. BCR is defined as a relapse of prostate cancer, the most important indicator of recurrence is the specific antigen (PSA $>0.2 \mathrm{ng} / \mathrm{mL}$ ) after surgery $[13,16,17]$. Dalela, et al. in his randomized study reported that the probability of BCRFS was 0.91 (95\% $\mathrm{Cl} 0.83-1.0)$ for anterior versus 0.91 (0.83-0.99) for posterior RARP $(p=0.5)$ [18]. The incidence of PSM after $\mathrm{RP}$ depends on several issues, including tumor biology, patient characteristics, pathological evaluation method, and surgeon's experience, surgical and potentially surgical technique.

Our current meta-analysis revealed that the PSM were not significantly different between the C-RARP and the RS-RARP. Similarly, in a series of two case studies of RS-RARP, one by Galfano in [8] and the other by Santok and colleagues [19] that contain 200 and 294 cases respectively, PSM was found in $25.5 \%$ and $22.7 \%$, respectively. On the other hand, some authors have recognized values of PSM slightly higher in those who received RS compared to the conventional technique.

In the work of Lim, et al. [12] there were no differences in the rates of (PSM) there was more tumor extension in the anterior face in the RS-RARP technique compared with the C-RARP, in the subgroup analysis PT2 and pT3 there were no significant differences in the PSM rates. Eden, et al. Obtained results in which [20] PSM were the same for RS-RARP and C-RARP, the location of anterior PSM was higher but without significant value $(5.0 \%$ vs. $0.0 \%, p=0.49)$.

Based on these previous results, some investigators have advised against performing RS-RARP if the tumor 
involves the anterior region. With the respect of Menon [11] deserves it was found that there were no statistically significant differences between the 2 groups in the proportion of non-focal margins, which was $11.7 \%$ for RS-RARP and $8.3 \%$ for C-RARP. In the work of Chang [21] the margin involved in the RS-RARP group was 9 patients $(23.3 \%)$ compared to 8 patients of C-RARP $(26.7 \%$, $p=0.261$ ), without significant differences. These negative results could be related to the learning curve of this novel approach and it's expected to be improved over time as shown in C-RARP. However, some other investigators did not report these similar findings in their studies [22].

\section{Continence}

RS-RARP allows a rapid recovery of continence; this was shown as a great advantage that was described in most studies of RS-RARP vs. C-RARP. In the RS-RARP group compared to the C-RARP After surgery, the general recovery of continence was quicker in the RS-RARP approach, we must take into account the maximum preservation made of the structures in the anterior part of the prostate gland such as puboprostatic fascia pubovesical ligaments, NVB and Santorini's Plexus (DVC) [23]. These structures participate in an important way, helping the recovery of continence, including the reconstruction of the Retzius space after transperitoneal RARP as stated by Ghanem, which found a faster early continence rate on their cases.

Compared to $48 \%$ in the C-RARP, $71 \%$ of the men subjected to RS-RARP were continent at the first week of removal of the probe $(p=0.01)$ [18] with respect to the study de Ghanem, et al. The continence rate was high in both groups ( $96 \%$ for RS-RARP and $94 \%$ for C-RARP) [9]. However, continence recovery (i.e., 0 pads) was significantly faster in the RSR-RARP group compared to the control group $(p=0.01)$. Also in the work of Menon [11] At 6 and 12 months after surgery $98.3 \%$ of men treated with the technique of RS-RARP and $93.3 \%$. Treaties with the C-RARP were socially continents [11]. Concerning the work of Lim, et al. East reports that the immediate continence was significantly higher at 7 days in the group of RS-RARP, although no significant difference were reported at the end of 1 year.

The preservation of Retzius space structures could lead to a more anatomical and physiological environment for the previous individual continent status.

\section{Potency recovery}

The perceptions of preservation of the neurovascular bundles become more and more significant; preservation could partially affect the recovery from the emotional and psychological point of view of the patient [24]. The RARP that preserves the nerves involves different surgical techniques, such as intrafascial or interfascial, anterograde or retrograde approach; meanwhile, there is a lack of randomized controlled trials to define which technique is superior to another. The individual anatomical factors of patients can affect these techniques and approaches; therefore, the surgeon experience is important to decide which surgical technique is more suitable, always trying to reach for incremental nerve preservation [25].

In the work of Galfano [8], he reports that he is the only author who reports sexual function data in his second work of RS-RARP reports 7 powerful patients aged $<65$ years who underwent bilateral intrafascial nerve preservation.

In surgery, $40.4 \%$ of group 1 versus $40 \%$ of group 2 reached their first sexual intercourse within 1 month; at 1 year of follow-up, these figures had increased to $81 \%$ compared to $71 \%$, respectively ( $p=0.162$ ) [8]. In the work of Menon [11] with C-RARP vs. RS-RARP one year after surgery (69.2\% vs. $86.5 \%)$ they obtained sufficient erection for intercourse and (44.6\% vs. $44.1 \%)$ obtained in the SHIM score a score of 17 or more [11].

We can conclude that in the majority of studies used in our meta-analysis, the results regarding sexual potency are not well informed, and more clinical trials are needed to evaluate sexual function in this scenario.

\section{Conclusion}

The literature on RS-RARP has few well-designed studies and large participants where long follow-up is carried out. The access has proven to be safe and feasible although technically more challenging than the conventional technique and possibly requiring a longer learning curve. This meta-analysis shows that the RS-RARP and RSR-RARP compared to the conventional technique, achieves a faster continence, the complication rates and the PSMs have similar rates, and no conclusion regard erectile dysfunction could be done.

There are other predictors that can influence the functional and oncological results other than the technique used such as the patient's age, the preoperative IPSS and the SHIM score, the existence of diabetes mellitus, the surgeon's experience, the extent of the tumor and many others. The boundaries of observational studies, short follow-up, short study time and small data in our meta-analysis may avoid a definitive conclusion. Randomized future clinical trials are required to validate and confirm our suggestions.

\section{Acknowledgements}

To our families.

\section{Contributions of Authors}

WEPG, MTM and PAMM: Conceptualization, data management, formal analysis, research, methodology, project management, supervision, data presentation, manuscript preparation (development of the original 
draft), writing (revisions and editions).

MCB: conceptualization, data management, research, preparation of manuscript (development of the original draft), writing (revisions and editions).

PRA and LEP: Conceptualization, data management, research, preparation of manuscript (development of the original draft), writing (revisions and editions).

\section{Declarations of Interest}

No conflicts of interests.

\section{References}

1. Bell KJL, Del Mar C, Wright G, Dickinson J, Glasziou P (2015) Prevalence of incidental prostate cancer: A systematic review of autopsy studies. Int J Cancer 137: 1749-1757.

2. Ferlay J, Soerjomataram I, Dikshit R, Eser S, Mathers C, et al. (2015) Cancer incidence and mortality worldwide: Sources, methods and major patterns in GLOBOCAN 2012. Int J 136: E359-E386.

3. Agarwal G, Valderrama O, Luchey AM, Pow-Sang JM (2015) Robotic-assisted laparoscopic radical prostatectomy. Cancer Control 22: 283-290.

4. Patel VR, Abdul-Muhsin HM, Schatloff O, Coelho RF, Valero $R$, et al. (2011) Critical review of "pentafecta" outcomes after robot-assisted laparoscopic prostatectomy in high-volume centres. BJU Int 108: 1007-1017.

5. Galfano A, Ascione A, Grimaldi S, Petralia G, Strada E, et al. (2019) A new anatomic approach for robot-assisted laparoscopic prostatectomy: A feasibility study for completely intrafascial surgery. Eur Urol 58: 457-461.

6. Freire MP, Weinberg AC, Lei Y, Soukup JR, Lipsitz SR, et al. (2009) Anatomic bladder neck preservation during robotic-assisted laparoscopic radical prostatectomy: Description of technique and outcomes. Eur Urol 56: 972-980.

7. Kaul S, Savera A, Badani K, Fumo M, Bhandari A, et al. (2006) Functional outcomes and oncological efficacy of Vattikuti Institute prostatectomy with Veil of Aphrodite nerve-sparing: An analysis of 154 consecutive patients. BJU Int 97: 467-472.

8. Galfano A, Di Trapani D, Sozzi F, Strada E, Petralia G, et al. (2013) Beyond the learning curve of the retzius-sparing approach for robot-assisted laparoscopic radical prostatectomy: Oncologic and functional results of the first 200 patients with $\geq 1$ Year of follow-up. Eur Urol 64: 974-980.

9. Abu-Ghanem Y, Dotan Z, Ramon J, Zilberman DE (2018) Retzius space reconstruction following transperitoneal laparoscopic robot-assisted radical prostatectomy: does it have any added value? J Robot Surg 12: 475-479.

10. http://www.annualreports.com/Company/intuitive-surgical-inc

11. Menon M, Dalela D, Jamil M, Diaz M, Tallman C, et al. (2018) Functional recovery, oncologic outcomes and postoperative complications after robot-assisted radical prostatectomy: An evidence-based analysis comparing the retzius sparing and standard approaches. J Urol 199: 1210-1217.

12. Lim SK, Kim KH, Shin T-Y, Han WK, Chung BH, et al. (2014) Retzius-sparing robot-assisted laparoscopic radical prostatectomy: Combining the best of retropubic and perineal approaches. BJU Int 114: 236-244.

13. Heidenreich A, Bastian PJ, Bellmunt J, Bolla M, Joniau S, et al. (2014) EAU guidelines on prostate cancer. Part II: treatment of advanced, relapsing, and castration-resistant prostate cancer. Eur Urol 65: 467-479.

14. Patel VR, Coelho RF, Rocco B, Orvieto M, Sivaraman A, et al. (2011) Positive surgical margins after robotic assisted radical prostatectomy: A multi-institutional study. J Urol 186: 511-517.

15. Yossepowitch O, Briganti A, Eastham JA, Epstein J, Graefen $M$, et al. (2014) Positive surgical margins after radical prostatectomy: A systematic review and contemporary update. Eur Urol 65: 303-313.

16. Sooriakumaran $P$, John $M$, Wiklund $P$, Lee $D$, Nilsson $A$, et al. (2011) Learning curve for robotic assisted laparoscopic prostatectomy: a multi-institutional study of 3794 patients. Minerva Urol Nefrol 63: 191-198.

17. Jo J-K, Hong S-K, Byun S-S, Zargar H, Autorino R, et al. (2017) Positive surgical margin in robot-assisted radical prostatectomy: correlation with pathology findings and risk of biochemical recurrence. Minerva Urol Nefrol 69: 493500.

18. Dalela D, Jeong W, Prasad M-A, Sood A, Abdollah F, et al. (2017) A pragmatic randomized controlled trial examining the impact of the retzius-sparing approach on early urinary continence recovery after robot-assisted radical prostatectomy. Eur Urol 72: 677-685.

19. Santok GD, Abdel Raheem A, Kim LHC, Chang K, Lum TGH, et al. (2017) Perioperative and short-term outcomes of Retzius-sparing robot-assisted laparoscopic radical prostatectomy stratified by gland size. BJU Int 119: 135-141.

20. Eden CG, Moschonas D, Soares R (2018) Urinary continence four weeks following Retzius-sparing robotic radical prostatectomy: The UK experience. J Clin Urol 11: 15-20.

21. Chang L-W, Hung S-C, Hu J-C, Chiu K-Y (2018) Retzius-sparing robotic-assisted radical prostatectomy associated with less bladder neck descent and better early continence outcome. Anticancer Res 38: 345-351.

22. Sayyid RK, Simpson WG, Lu C, Terris MK, Klaassen Z, et al. (2017) Retzius-Sparing robotic-assisted laparoscopic radical prostatectomy: A safe surgical technique with superior continence outcomes. J Endourol 31: 1244-1250.

23. Asimakopoulos AD, Miano R, Galfano A, Bocciardi AM, Vespasiani G, et al. (2015) Retzius-sparing robot-assisted laparoscopic radical prostatectomy: Critical appraisal of the anatomic landmarks for a complete intrafascial approach. Clin Anat 28: 896-902.

24. Walsh PC, Donker PJ (2017) Impotence following radical prostatectomy: Insight into etiology and prevention. J Urol 197: S165-S170.

25. Tavukçu HH, Aytac O, Atug F (2016) Nerve-sparing techniques and results in robot-assisted radical prostatectomy. Investig Clin Urol 57: S172-S184.

26. Marcos Tobias-Machado (2017) Video endoscopic inguinal lymphadenectomy (VEIL): Is a new standard ready to be accepted? BJUI 119: 504-505. 
Supplementary Table 1: Search strategies for full-text articles.

\section{Searches \\ PubMed}

(((Retzius-sparing robot assisted radical prostatectomy OR posterior RARP OR Retzius-sparing approach OR RS-RARP)) AND (Retzius-sparing robot assisted radical prostatectomy OR anterior RARP OR anterior approach OR conventional robot assisted radical prostatectomy OR C-RARP)) AND (Continence recovery OR Overall continence recovery OR Completely dry OR Time to urinary continence recovery) $=13$

(((Retzius-sparing robot assisted radical prostatectomy OR posterior RARP OR Retzius-sparing approach OR RS-RARP)) AND (Retzius-sparing robot assisted radical prostatectomy OR anterior RARP OR anterior approach OR conventional robot assisted radical prostatectomy OR C-RARP)) AND (Positive marginal margins OR surgical margins) $=10$

(((Retzius-sparing robot assisted radical prostatectomy OR posterior RARP OR Retzius-sparing approach OR RS-RARP)) AND (Retzius-sparing robot assisted radical prostatectomy OR anterior RARP OR anterior approach OR conventional robot assisted radical prostatectomy OR C-RARP)) AND (Complication rates OR Claiven and Dindo classification) $=6$

(((Retzius-sparing robot assisted radical prostatectomy OR posterior RARP OR Retzius-sparing approach OR RS-RARP)) AND (Retzius-sparing robot assisted radical prostatectomy OR anterior RARP OR anterior approach OR conventional robot assisted radical prostatectomy OR C-RARP)) AND (International Prostate Symptom Score OR IPSS score or IPSS urinary function score OR IPSS) $=2$

(((Retzius-sparing robot assisted radical prostatectomy OR posterior RARP OR Retzius-sparing approach OR RS-RARP)) AND (Retzius-sparing robot assisted radical prostatectomy OR anterior RARP OR anterior approach OR conventional robot assisted radical prostatectomy OR C-RARP)) AND Sexual potency recovery $=1$

\section{EMBASE}

\#1 (('retzius sparing' AND ('robot'/exp OR robot) AND assisted AND ('radical'/exp OR radical) AND ('prostatectomy'/exp OR prostatectomy) OR posterior) AND rarp OR 'retzius sparing') AND approach OR 'rs rarp'

\#2 ((('retzius sparing' AND robot AND assisted AND radical AND prostatectomy OR anterior) AND rarp OR anterior) AND approach OR conventional) AND robot AND assisted AND radical AND prostatectomy OR 'c rarp'

\#3 (((continence AND recovery OR overall) AND continence AND recovery OR completely) AND dry OR time) AND to AND urinary AND continence AND recovery

\#4 (positive AND marginal AND margins OR surgical) AND margins

\#5 (complication AND rates OR claiven) AND dindo AND classification

\#6 ((international AND prostate AND symptom AND score OR ipss) AND score OR ipss)

AND urinary AND function AND score OR ipss

\#7 sexual AND potency AND recovery

\#1 AND \#2 AND \#3= 5

\#1 AND \#2 AND \#4= 14

$\# 1$ AND \#2 AND \#5= 1

$\# 1$ AND \#2 AND \#6= 6

\#1 AND \#2 AND \#7= 1

COCHRANE (OVID)

Retzius-sparing robot assisted radical prostatectomy $=12$

Google Scholar

Retzius-sparing robot assisted radical prostatectomy $=149$

Gross: 212

Net, after duplicates removal and first broad cut: 178 